\title{
Mapping Correlation Dimension along the Wall Region of a High-Flux Gas-Solid Riser Using Embedded Solid Concentration Time Series
}

\author{
Justin M. Jeremiah1', Samwel V. Manyele1, Abraham K. Temu'1, Jesse-X. Zhu² \\ ${ }^{1}$ Department of Chemical and Mining Engineering, University of Dar es Salaam, Dar es Salaam, Tanzania \\ ${ }^{2}$ Department of Chemical and Biochemical Engineering, University of Western Ontario, London, Canada \\ Email: jeremiahjmj@udsm.ac.tz,smanyele@udsm.ac.tz, atemu@udsm.ac.tz,jzhu@uwo.ca
}

How to cite this paper: Jeremiah, J.M. Manyele, S.V., Temu, A.K. and Zhu, J.-X. (2018) Mapping Correlation Dimension along the Wall Region of a High-Flux Gas-Solid Riser Using Embedded Solid Concentration Time Series. Engineering, 10, 655-679. https://doi.org/10.4236/eng.2018.1010048

Received: May 30, 2018

Accepted: October 15, 2018

Published: October 18, 2018

Copyright $\odot 2018$ by authors and Scientific Research Publishing Inc. This work is licensed under the Creative Commons Attribution-NonCommercial International License (CC BY-NC 4.0). http://creativecommons.org/licenses/by-nc/4.0/

\begin{abstract}
Analysis of the entrance and wall dynamics of a high-flux gas-solid riser was conducted using embedded solid concentration time series collected from a $76 \mathrm{~mm}$ internal diameter and $10 \mathrm{~m}$ high riser of a circulating fluidized bed (CFB) system. The riser was operated at 4.0 to $10.0 \mathrm{~m} / \mathrm{s}$ air velocity and 50 to $550 \mathrm{~kg} / \mathrm{m}^{2} \mathrm{~s}$ solids flux of spent fluid catalytic cracking (FCC) catalyst particles with $67 \mu \mathrm{m}$ mean diameter and density of $1500 \mathrm{~kg} / \mathrm{m}^{3}$. Data were analyzed using prepared FORTRAN 2008 code to get correlation integral followed by determination of correlation dimensions with respect to the hyperspherical radius and their profiles, plots of which were studied. It was found that correlation dimension profiles at the centre have single peak with higher values than the wall region profiles. Towards the wall, these profiles have double or multiple peaks showing bifractal or multifractal flow behaviors. As the velocity increases the wall region profiles become random and irregular. Further it was found that, as the height increases the correlation dimension profiles shift towards higher hyperspherical radius at the centre and towards lower hyperspherical radius in the wall region at $r / R=0.81$. The established method of mapping correlation dimension profiles in this study forms a suitable tool for analysis of high-flux riser dynamics compared to other analyses approaches. However, further analysis is recommended to other gas-solid CFB riser of different dimensions operated at high-flux conditions using the established method.
\end{abstract}

\section{Keywords}

Correlation Integral, Mapping Correlation Dimension, High-Flux Gas-Solid Riser, Embedded Solid Concentration Time Series 


\section{Introduction}

The gas-solid circulating fluidized bed (CFB) systems have been shown to be complex and largely governed by the non-linear relationships. The solid concentration or voidage and pressure time series from these fluidized beds systems exhibit the characteristics of low dimensional deterministic chaos [1]. Studying the dynamics of such fluidized bed systems requires non-linear techniques like chaos analysis which involves a multi-dimensional study approach in systems exhibiting complex and non-linear dynamics [2] [3] [4]. Chaos analysis of solid concentrations and pressure fluctuation signals from circulating fluidized bed riser are widely applied in studying systems dynamics due to their ability to account for the complex and non-linear nature of the gas-solid flow in fluidized beds [3] [5].

Correlation dimension is one of the chaos analysis parameters which is widely used to account for the complexity of the gas-solid flow systems in riser reactors [3]. It is determined as the slope of the linear part of the correlation integral versus hyperspherical radius curve using the Grassberger and Procaccia algorithm [3]. Once determined, it is related to the gas-solid flow characteristics in order to establish the system flow dynamic behaviours. Several studies have shown the presence of more than one correlation dimension at the correlation integral versus hyperspherical radius curve [3]. However, only one correlation dimension is mostly used to characterize the gas-solid flow behaviours in the riser [3]. The presence of multiple correlation dimensions along the correlation integral versus hyperspherical radius curve and their use in characterizing the complexity of gas-solid flow in the entrance section and wall region as a result of change in the operating conditions and locations within the high-flux gas-solid riser has not been studied.

This study investigated the flow dynamics behaviors of the gas-solids suspensions flow in the riser reactor using the measured solid concentration time series signals. In particular, this paper presents chaos analysis of the temporal variations of solid concentration in the entrance section, flow development section, developed flow sections and in the wall region of a high flux gas-solid riser using correlation dimension parameter.

This paper makes the use of the fact that the correlation dimension varies along the correlation function as the hyperspherical radius $(r)$ is changed across the attractor. While only the maximum value is normally reported by different researchers [3] [6] [7] [8], in this paper, a mapping technique is used by computing the correlation dimension along the correlation integral. The maximum value which appears as a peak on the profile of correlation dimension versus $\ln (r)$, defined in this study as correlation dimension profiles, is not only reported but also plotting the profiles of correlation dimension (that is, mapping of the correlation dimension) along the $\ln (r)$ axis across the reconstructed attractor is used to study the chaotic behavior of the gas-solid high-flux riser. The signals from the wall region along different riser sections (entrance, flow development 
and fully developed flow sections) were used to accomplish the study.

\section{Literature Review}

\subsection{Chaos Analysis of Non-Linear System}

Chaos analysis applies to non-linear and deterministic systems whose behavior can in principle be predicted for a while and then appears to become random. Studies show that, dilute gas-solid suspension flow is predictable, until when clusters form and pass across a specific point, events which appear at short intervals as depicted by cluster frequency [9]. The time for which prediction of the chaotic behaviour can be made depends on the tolerated uncertainty, the accuracy of measurements and the time scale referred to as the Lyapunov time, for instance, the sampling frequency, depending on the dynamics of the system [9]. Further, it is shown that in chaotic systems, the uncertainty in a forecast increases exponentially with elapsed time measured closely by a chaotic parameter called Kolmogorov entropy. Therefore, if the time of forecast is doubled, this result to the squares of the uncertainties predicted which further implies that the significant prediction can be made in two or three times the Lyapunov time not more. The system is said to be random at the point when the meaningful and significant predictions cannot be made [9] [10].

Literatures show that non-linear time series analyses techniques are founded on various theories of dynamical system of which its time of evolution is expressed in some phase space [11]. Thus, analysis of such system can be achieved through representation of the phase space. If the current state of the dynamical system can be accurately identified and fixed then the future states can be identified through systematic studies of the phase space where all states of such system are presented [11].

Thus, being one of the non-linear techniques, chaos analysis is achieved by first reconstructing the attractor in the phase space or state space [3]. A state space is a space spanned by dependent variables of a given dynamical system in which all possible states of a system are represented, with each possible state of the system corresponding to one unique point in the phase space [5] [11]. In order to accomplish the analysis, an attractor is reconstructed in a phase space from which invariant parameters are determined and used to study the system dynamics [3] [6]. An attractor is defined as a geometrical structure which is set in a phase space where trajectories finally settles down [2] [3] [4]. Chaos analysis uses parameters such as Correlation dimension, Kolmogorov entropy, fractal dimension, Lyapunov exponent; Hurst rescaled range, Hilbbert-Huang transform, etc. However, of these parameters, the most commonly parameters used to characterize the attractor are the correlation dimension and the Kolmogorov entropy [1] [3] [6] [7].

The state of the gas-solid dynamical system in a CFB riser can be defined by a time series of one of the system's operating conditions or parameters such as the voidage or solid concentrations and pressure fluctuations. This is referred to as time series of single variable. It is from such a time series of a single measured 
parameter where reconstruction of a phase space or state-space is done through embedding method which utilizes time delay and embedding dimension [1]. The system's dynamics is then described from the reconstructed phase space from which chaotic parameters are computed and then used as a quantitative measures of a chaotic behavior of a particular system [5] [11].

The reconstructed phase space by using a single variable signal to represent other true variables, through time lag which is applied to the single variable signal, mimics the true phase space which is created from the true process variables. The CFB riser, for instance, is operated under various true variables such as gas velocity and solids flux; the measured solid concentrations, $\varepsilon_{s}(t)$, can be used to reconstruct the true attractor by using the time delay. The number of variables that affect the system can be estimated from the reconstructed phase space and the determined system invariants such as correlation dimensions. However, in most studies once the correlation dimension is established, and the variation of such a dimension with operating conditions and spatial location is known, the outcome is taken to be a sufficient description of the complex system.

\subsection{Determination of Correlation Dimension}

Correlation dimension is a measure of the fractal dimension of the time series, which express the complexity that quantifies or gives the characteristics of the geometry and shape of the attractor. It measures the scaling of the points along trajectories on the attractor, and tells to which exponential law these points follow. The higher the correlation dimension, the more the complex microscopic flow dynamics and vice versa in relation to the time scale. Microscopic level is depicted by smaller volume of measurement point used, especially when optic fibers are used, as compared to pressure drops across a riser section which can be referred to as macro scale. The values of the correlation dimension are commonly obtained by first plotting the graph of $\log (C r)$ versus $\log (r)$ which shows the linear dependence in some ranges of $r$ values. Correlation dimension estimator of the attractor is the slope of the scaling region of the correlation integral, $C(r)$, versus hypersphere radius, $r$, [6] [12] [13]. However, in this study the graph of correlation dimension versus hyperspherical radius, defined in this study as the correlation dimension profiles, has been utilized to compare the flow dynamics of a high flux CFB riser, The shape of the correlation dimension profile, its location and the magnitude of the peak value or values in case of multiple peaks, forms an investigation tool which has been used in this study.

The process of determining correlation dimension involves first computing the correlation sum which counts the vector pairs $\left(X_{\mathrm{i}}, X_{\mathrm{j}}\right)$ whose distance is smaller than the selected hyperspherical radius, $r$, as given in Equation (1). The counting is started by letting the count $i$ constant, and then traversing all counts $j$ to the total number of data points, $N-1$, in the time series before $i$ is also changed up to $N$ in this case. When the total data points approaches to infinity, i.e., $N \rightarrow \infty$, then the value of $N(N-1)$ approaches to $N^{2}$. The correlation sum, 
$C(r)$, is determined as shown in Equation (1) [6].

$$
C_{m}(r)=\lim _{N \rightarrow \infty} \frac{1}{N^{2}} \sum_{i=1}^{N} \sum_{j=i+1}^{N} \Theta\left(r-\left[\sum_{k=1}^{m}\left(\varepsilon_{s}(i, k)-\varepsilon_{s}(j, k)\right)^{2}\right]^{\frac{1}{2}}\right)
$$

where $\varepsilon_{s}(i, k)$ and $\varepsilon_{s}(j, k)$ represents the vector points of the solid concentration data signals and $\Theta(u)$ is the Heaviside step function, for which:

$$
u=r-\left[\sum_{k=1}^{m}\left(\varepsilon_{s}(i, k)-\varepsilon_{s}(j, k)\right)^{2}\right]^{\frac{1}{2}} \text { and } \Theta(u)=\left\{\begin{array}{l}
1, \text { if } u \geq 0 \\
0, \text { if } u<0
\end{array}\right.
$$

Thus, the correlation sum, $C(r)$, is a cumulative distribution function (CDF) which reflects the distribution probability of the vector distances which are less than $r$ between pairs of points on the chaotic attractor [14]. Studies show that in the limit of an infinite amount of data points $(N \rightarrow \infty)$ and for small radius, $r$, such that $r_{\min }<r<r_{\max }$ then $C(r)$ scale like a power law. Further, if the attractor characterises the time series of a single dynamical variable, in the positive ranges of the hyperspherical radius, $r$, the correlation sum, $C(r)$, is related to the hyperspherical radius, $r$, by the power law as per Equation (2) [15]:

$$
C(r) \propto r^{D}
$$

where $D$ is the characteristic parameter of an attractor referred as correlation dimension which is the. Thus, when there is an attractor in the phase space, the relationships can be presented as $C(r) \sim r^{D}$, where $D$ is the topological dimension of the attractor. The topological dimension can be regarded as local correlation dimension determined at every short distances, $r$. The dimension, $D$, is also equal to the topological dimension $D$ of the attractor if the system dynamics is classified as periodic or quasi-periodic. Consequently, the fractal dimension $D$ is not an integer if the system is chaotic and the attractor is a strange attractor. Thus according to the power law property, basing on the behavior of a correlation sum, a dimension value $D$ can be defined as [14] [15]:

$$
D(r)=\lim _{r \rightarrow 0} \lim _{N \rightarrow \infty} \frac{d[\log C(r)]}{d[\log (r)]}
$$

Since the number of points is fixed during computations of correlation sum, $C(r)$, then Equation (4) simplifies to Equation (5):

$$
D(r)=\lim _{r \rightarrow 0} \frac{d[\log C(r)]}{d[\log (r)]}
$$

Using the scaling region, $D$ is the slope of $\ln (C r)$ versus $\ln (r)$ plot. The slope $D$ of the linear part of the log-log curve provides all necessary information for characterizing the attractor [16]. The slope is generally estimated by a least square fit of a straight line over a certain range of $r$ called the scaling region, which is a radius interval of sufficient length at small $r$ where $D(r)$ remains approximately constant and regards this value as an estimate of $D(r)$ [17]. There are various methods established to identify the scaling region. However, the region is gener- 
ally identified by visual inspection though its scale or its upper and lower limit may differ which may result in variation in the calculated correlation dimension [14]. If the resulting estimate is non-integer then this shows that the attractor is a fractal [18]. The length of the point interval within $r$ which should be used to determine the correlation dimension, has been deal in this paper, by computing the values of $D$ for a varying number of points along the correlation integral based on which a three-point interval was selected.

\subsection{Determination of Minimum Embedding Dimension and Time Delay}

Chaos analysis begins by reconstruction of the phase space through embedding techniques [3]. This achieved by first establishing the embedding parameters, the minimum embedding dimension and the time delay. The minimum embedding dimension is the dimension where a time delay reconstruction of the system's phase space provides a necessary number of coordinates to unfold the dynamics from overlaps on itself caused by projection [19]. According to Taken's theorem, to characterize a dynamic system with an attractor dimension $D$, an $m$-dimensional phase space for which $m \geq 2 D+1$ is required. There are methods which are used to determine the embedding dimension, $m$ [15] [18] [19] [20] [21] [22]. In this study, the minimum embedding dimensions was determined by saturation of correlation dimension as the embedding dimensions increases. Also there are methods used to determine the time delay [15]. In this study the time delay, $\tau$, for embedding the solids concentration was estimated by use of correlation dimension versus time delay curve, determined by embedding solid concentration data from high-flux gas-solid riser at different time delay.

\section{Methodology}

\subsection{Data Collection}

Data were collected from a CFB system shown in Figure 1 with a twin-riser having 76 and $203 \mathrm{~mm}$ internal diameters and $10 \mathrm{~m}$ high operated at 50 to 550 $\mathrm{kg} / \mathrm{m}^{2} \mathrm{~s}$ solids flux and 4.0 to $10.0 \mathrm{~m} / \mathrm{s}$ gas velocity. In particular $U_{g}=5.5 \mathrm{~m} / \mathrm{s}, 8.0$ $\mathrm{m} / \mathrm{s}$ and $10 \mathrm{~m} / \mathrm{s}$ were used in this study. Fluid catalytic cracking catalyst particles with $67 \mu \mathrm{m}$ mean diameter and density of $1500 \mathrm{~kg} / \mathrm{m}^{3}$ were used. A $70 \%$ to $80 \%$ humid air was used for transporting the solid particles. Signals were sampled from eight (8) axial levels (i.e., $Z=0.98,1.52,2.73,3.96,5.13,6.34,8.74$, and 9.42 $\mathrm{m}$ ) and 11 radial points (i.e., $r / R=0.00,0.16,0.38,0.50,0.59,0.67,0.74,0.81$, $0.87,0.92$, and 0.98 ) at each level where $r / R$ is the normalized radial distances from the center to the wall of the riser. To each point, 29,100 data points of solid concentration were sampled in 30 seconds using optical fiber probe at $970 \mathrm{~Hz}$.

\subsection{Determination of Minimum Embedding Dimension and Time Delay}

The embedding dimension was determined by the method of saturation of correlation dimension. The maximum correlation dimension was determined at 


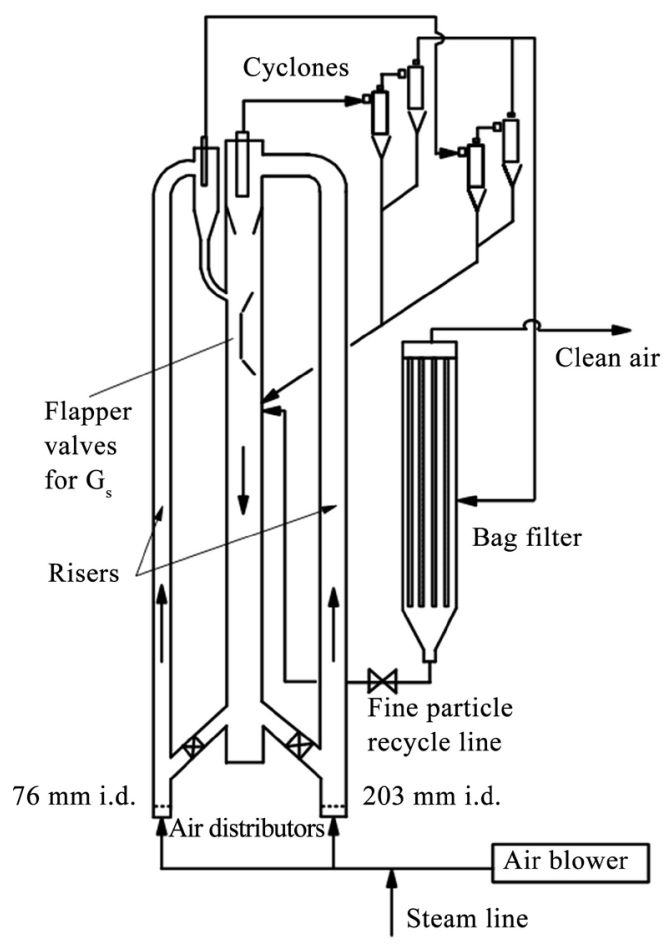

Figure 1. The circulating fluidized bed with two riser system.

different embedding dimensions and the profile drawn. The minimum embedding dimension was determined at the point where maximum correlation dimension saturate. The time delay was determined from the maximum correlation dimension versus time delay profile. The time delay $(\tau)$ can be any value as it is not going to change the attractor of the system. But it decides the independency of the coordinates. The time delay that keeps the coordinates more time independent is desirable. Too large values cause loss of information contained in the data and too temporally close vectors become rather remote, giving rise to uncertainties i.e. large delays lead to vectors whose components are (almost) uncorrelated and the data are thus randomly distributed in the embedding space. On the other hand, small time delay yields correlated vector (crossing among the trajectories) elements. Figure 2 shows the plot of the maximum correlation dimension, max. $D_{3}$, against the embedding dimension, $m$, and the time delay, $\tau$, at $Z=1.52 \mathrm{~m}$ for $U_{\mathrm{g}}=5.5 \mathrm{~m} / \mathrm{s}$ and $G_{\mathrm{s}}=300 \mathrm{~kg} / \mathrm{m}^{2} \mathrm{~s}$. From Figure 2 the saturation of the maximum correlation dimension starts at $m=15$, for $r / R=$ 0.98 which was determined as the sufficient number of embedding dimension for this study.

The delay time, $\tau$, was estimated from point where the curve gives the higher correlation dimension after the first minimum value. From Figure 2 the first minimum give the delay time, $\tau=5$ and the higher value after the first minimum gives delay time, $\tau=6$ for $r / R=0.98$. Thus, the delay time, $\tau=6$ was found sufficient and chosen to study the dynamics in the entrance and the wall region of the high flux gas-solid riser. 


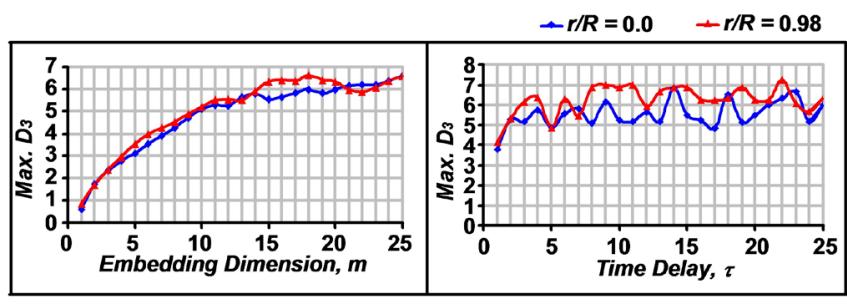

Figure 2. Variation of correlation dimension with number of embedding dimensions and delay time at $Z=1.52 \mathrm{~m}$ for $U_{\mathrm{g}}=5.5 \mathrm{~m} / \mathrm{s}$ and $G_{\mathrm{s}}=300 \mathrm{~kg} / \mathrm{m}^{2}$ s.

\subsection{Determination of Correlation Dimension}

Determination of correlation dimensions $\left(D^{\prime} s\right)$ were achieved by first computing correlation integrals, $C(r)$, at various hyperspherical radius, $r$, by applying Equation (1) incodes prepared in FORTRAN 2008. Correlation dimensions were then determined as the slope of the correlation integral, $\ln (C r)$, versus hypersphere radius, $\ln (r)$, which were estimated between point intervals of three to nine steps. Slope was determined as the ratio of change in $\ln (C r)$ to the change in $\ln (r)$, to get $D_{3}, D_{4}, \ldots, D_{9}$ using Equation (4). Determined correlation dimensions were plotted to get $D^{\prime} s$ versus $\ln (r)$ plots which were assessed. The $D_{3}$ versus $\ln (r)$ plots gave the maximum value at each signal and hence these plots were used for further analysis, Maximum values were also determined, plotted and analysed. This method is established in this study to analyse the high-flux gas-solid CFB riser.

\subsection{Determination of Correlation Dimension $\left(D_{3}\right)$ Profiles}

Given the correlation function, $C(r)$, in discrete form, values of which were established at several values of hyperspherical distance, $r$, the first derivative of the $\ln (C r)$ function with respect to $\ln (r)$ yields the correlation dimension function of $\ln (r)$. Thus, the $D_{3}$ values were obtained as per equation:

$$
D_{3}(\ln (r))=\left.\frac{d[(\ln C(r))]}{d[(\ln (r))]}\right|_{n} ^{n+2}
$$

This is the slope determined at intervals of consecutive three (3) points. The values of $D_{3}$ corresponding to the $\mathrm{n}^{\text {th }}$ value of $\ln (r)$, a plot of which is defined in this study as $D_{3}$ profile. Profile of $D$ from different intervals, 4 to 9 , along $\ln (r)$ did not yield the desired maximum values, hence the study focused on $D_{3}$ values or $D_{3}$ profiles. The use of these profiles to study the flow dynamics in the high-flux gas-solid riser is also established in this study.

\section{Results and Discussion}

\subsection{Maximum Correlation Dimension for High Flux Data}

Figure 3 presents the correlation dimension plots at the centre and the wall in the entrance, flow development and the top sections of the riser operated at $U_{g}=$ $8 \mathrm{~m} / \mathrm{s}$ and $G_{s}=300 \mathrm{~kg} / \mathrm{m}^{2} \mathrm{~s}$. Correlation dimension can be determined from the 


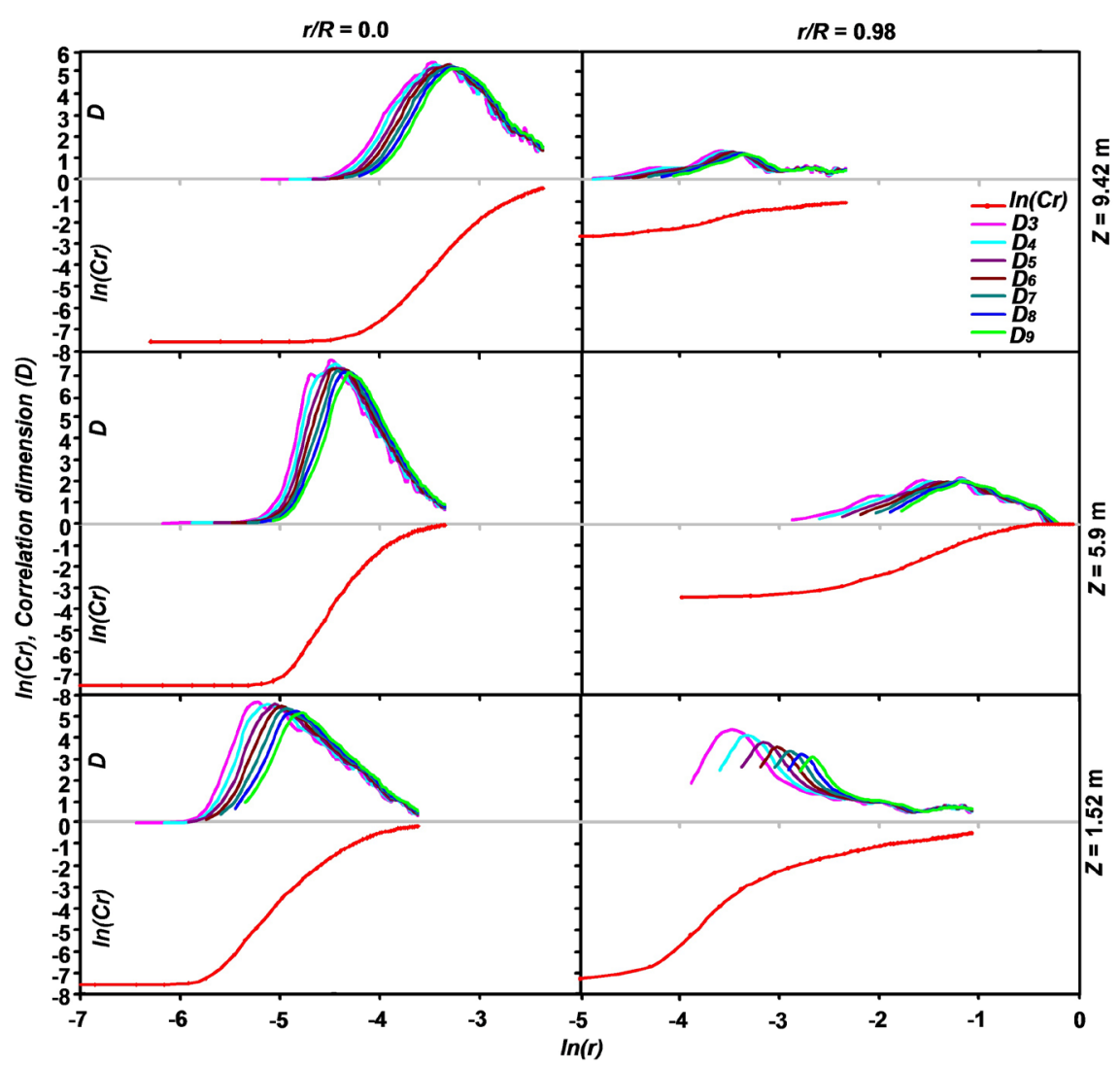

Figure 3. Maximum correlation dimension plots for high flux data at different axial locations.

slope of $\ln (C r)$ and $\ln (r)$ plot as per Equation (5). The question of how many steps to use in the determination of slope is answered in Figure 3. Depending on the number of steps for slope determination, different values of $D$ were obtained. According to Grassberger and Procaccia [8], the maximum value is taken as $D$. The value of $D$ for any number of steps shows a maximum value or peak, that is, the profile has a peak or peaks where the maximum value is taken as a correlation dimension. This maximum varies with the number of steps as well. Figure 3 presents six plots of a series of correlation dimensions profiles from $D_{3}$ to $D_{9}$ (i.e., slopes obtained from three to nine steps) at the upper part and below there is a correlation integral profile from which the values of $D_{3}$ to $D_{9}$ were estimated. From $D_{3}$ to $D_{9}$, i.e., increasing the number of steps for determination of slope implies determination of the optimum distance, $r$, which brings the highest value of topological dimension, referred to as correlation dimension. Higher number of steps implies larger local $r, r \rightarrow \infty$. Comparing the values of $D_{3}$ to $D_{9}$, a three (3) steps correlation dimension (i.e., $D_{3}$ ) profile was found to give a maximum correlation dimension and thus used for further analysis of the correlation dimension. Since the single step of $r$, the smallest unit of $r$ is known setting $D_{3}$ implies $3 \Delta r$, which is well known. Thus, this study used $D_{3}$ values to estimate the correlation dimension for the given signals. It is noted also that across the whole correlation sum function, denoted as $\ln (C r)$ in Figure 3, there exists values of $D$ 
or $D_{3}$ ranging from zero to $D_{3}$, as $\ln (r)$ changes. Plots of $D_{3}$ versus $\ln (r)$ form different profiles of which characteristics are extensively studied in this work.

The correlation dimension profiles, i.e., $D_{3}$ at the centre region $(r / R=0.0)$ have only one peak value on the $D_{3}$ profile in all axial levels as shown in the bottom $(Z=1.52 \mathrm{~m})$, middle $(Z=5.9 \mathrm{~m})$ and top $(Z=9.42 \mathrm{~m})$ sections. However, correlation dimension profiles for signals near the wall $(r / R=0.98)$ in all axial levels have multiple peaks for $D_{3}$ for the entrance $(Z=1.52 \mathrm{~m})$, flow development $(Z=5.9 \mathrm{~m})$ and fully developed flow $(Z=9.42 \mathrm{~m})$ sections.

According to Equation (4), the correlation dimension value is defined when $r$ $\rightarrow 0$ and $N \rightarrow \infty$. That is, at small values of $r$ obtained from correlation sum using large number of data points in the original time series.

The single peak values of the correlation dimension profiles at the centre in all axial levels indicate the uniform flow structure or low tendency to the formation of segregated flow or solid distribution fluctuations compared to the wall region, where the flow is characterized by the formation of dense phase such as particle aggregates or clusters in the dilute phase. The multiple peak values of correlation dimensions with varying magnitudes towards the wall as shown at $r / R=0.98$ in the entrance section $(Z=1.52 \mathrm{~m})$, flow development $(Z=5.9 \mathrm{~m})$ and top or fully developed $(Z=9.42 \mathrm{~m}$ ) sections indicates the formation of multifractal flow structures which is attributed to the segregated flows, i.e., particle aggregates with varying solid concentrations. From Figure 3 it can be further observed that the peak or maximum values of the correlation dimension at the centre are relatively higher than those close to the wall. This indicates that the flow behavior at the centre $(r / R=0.0)$ is characterized by faster changing particle-gas interactions due to high interactions between particles in the dilute gas-solid suspension as compared to low interactions in the wall region due to high solid concentration and dense aggregates of high momentum which limits particle's motions.

Further observation of Figure 3 shows a maximum correlation dimension values on $D_{3}$ profile. A maximum correlation dimension is a good indicator that can reveal the dynamical differences between various points or states of the system. Thus, since $D_{3}$ profile gives a maximum correlation dimension it is therefore, a good and strong indicator of the flow complexity, variations of which can provide details of the micro-flow structure of the gas-solid flow suspensions compared to the rest of $D$ values, that is, $D_{4}$ to $D_{9}$. Moreover, a three-step slope show good linear correlation compared to $D_{4}$ to $D_{9}$ due to small range/width of the scaling region observed when using solid concentration signals. It should also be noted that chaos analysis is about effect of small changes in the gas-solid flow on the dynamics of the system such as noted in instantaneous solid concentrations, $\varepsilon_{s}(t)$, thus $D_{3}$ being dependent on small changes in distances across the attractor, forms a good estimator of the dynamics of the riser. In this case, the correlation dimension, $D_{3}$, was selected to be used as a measure of the gas-solid flow behaviors in this study. 


\subsection{Correlation Dimension Profiles in the Wall-Region of the High Flux Riser}

Based on discussion above, and also from literature, correlation dimension is a strong tool which can be used to indicate the complexity of the gas-solid flow behavior in the CFB riser reactors. A higher correlation dimension is related to a more complex microscopic flow dynamics while a lower value (flatter peak) shows a less complex flow dynamics with low particle-particle, gas-particle and particle-wall interactions based on shorter time scales depicted by sampling frequency. Figures 4-6 show the plots of the correlation dimension, $D_{3}$, (referred to $D$ in figures) versus $\ln (r)$, at $U_{g}=5.5 \mathrm{~m} / \mathrm{s}, 8.0 \mathrm{~m} / \mathrm{s}$ and $10 \mathrm{~m} / \mathrm{s}$ respectively at $G_{s}=$ $300 \mathrm{~kg} / \mathrm{m}^{2} \mathrm{~s}$.

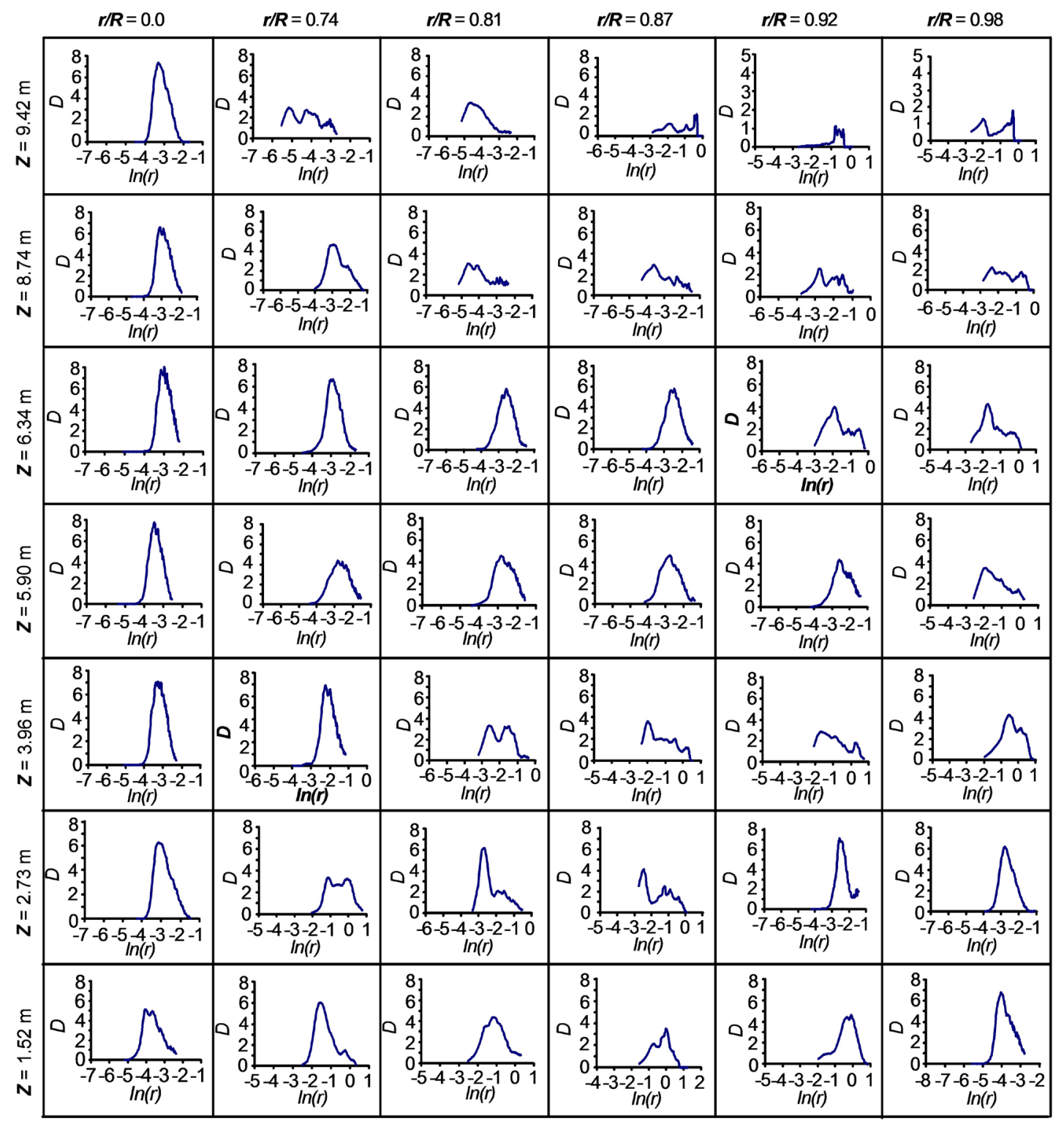

Figure 4. Mapping of correlation dimension along $\ln (r)$ axis for solid concentration signals from the wall-region of the high-flux riser at $U_{g}=5.5 \mathrm{~m} / \mathrm{s}, G_{s}=300 \mathrm{~kg} / \mathrm{m}^{2} \mathrm{~s}$. 


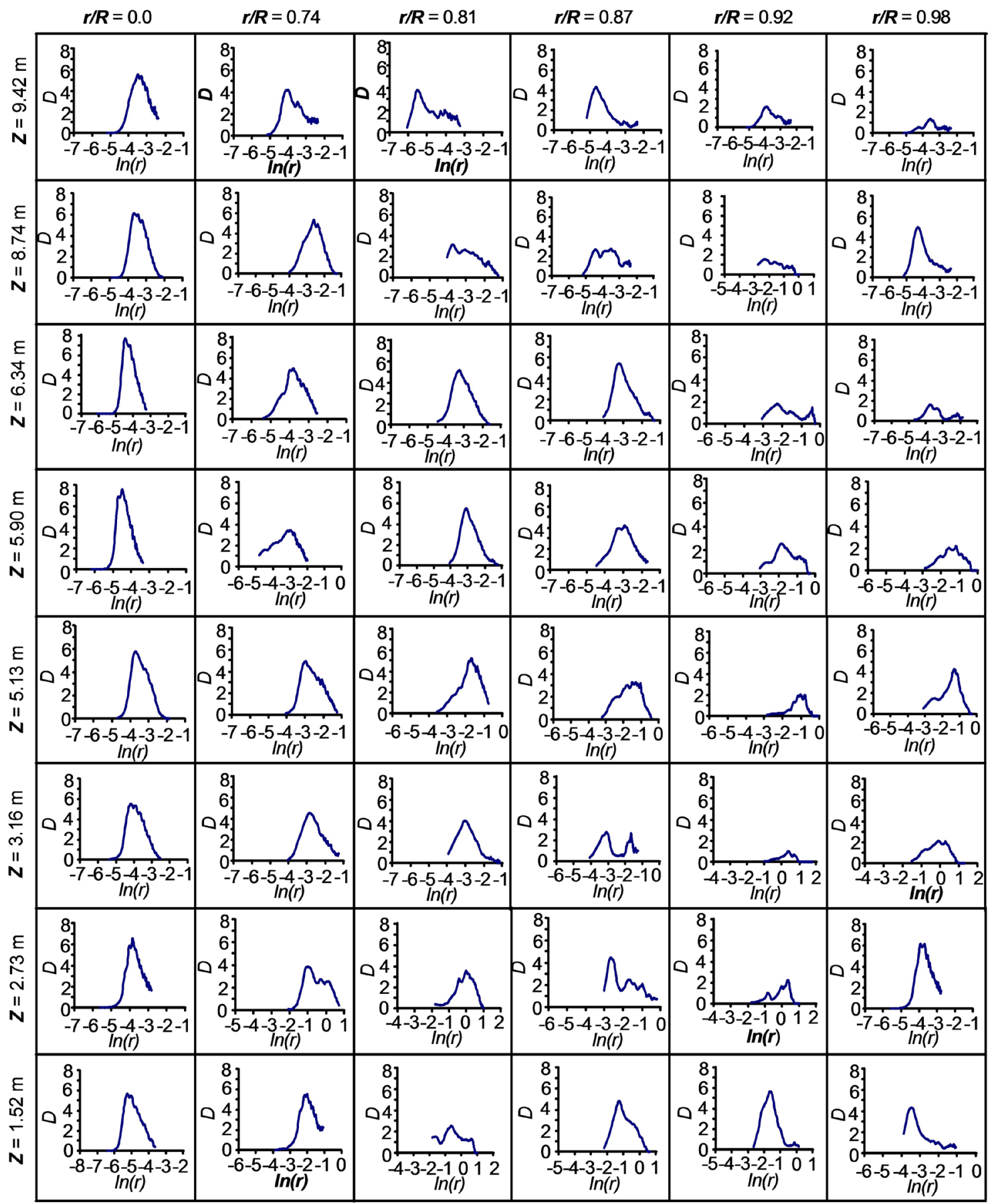

Figure 5. Mapping of correlation dimension along $\ln (r)$ axis for solid concentration signals from the wall-region of the high-flux riser at $U_{g}=8 \mathrm{~m} / \mathrm{s}, G_{s}=300 \mathrm{~kg} / \mathrm{m}^{2}$.

The $D_{3}$ plots show a peaked shape at the centre $(r / R=0.0)$ with highest values compared to the profiles in the wall region $(0.74 \leq r / R \leq 0.98)$. The Correlation dimension decreases from the centre of the riser towards the wall for all operating conditions investigated, i.e., $U_{\mathrm{g}}=5.5,8$ and $10 \mathrm{~m} / \mathrm{s}$ and $G_{\mathrm{s}}=300 \mathrm{~kg} / \mathrm{m}^{2} \mathrm{~s}$. Similar observation have been reported in the literature [3] where values of correlation dimension were higher at the centre and lowest values were observed 
near the wall $(r / R=0.87)$ where the solids concentration is high. The relatively higher values of the correlation dimensions at the center $(r / R=0.0)$ indicates more complex flow behaviors due to dilute flow which leads to high and faster interactions between particle-particle and particle-gas in the dilute solid-gas suspension compared to the wall region. The high degree of freedom leads to faster temporal variations of solid concentration in the centre of the riser.

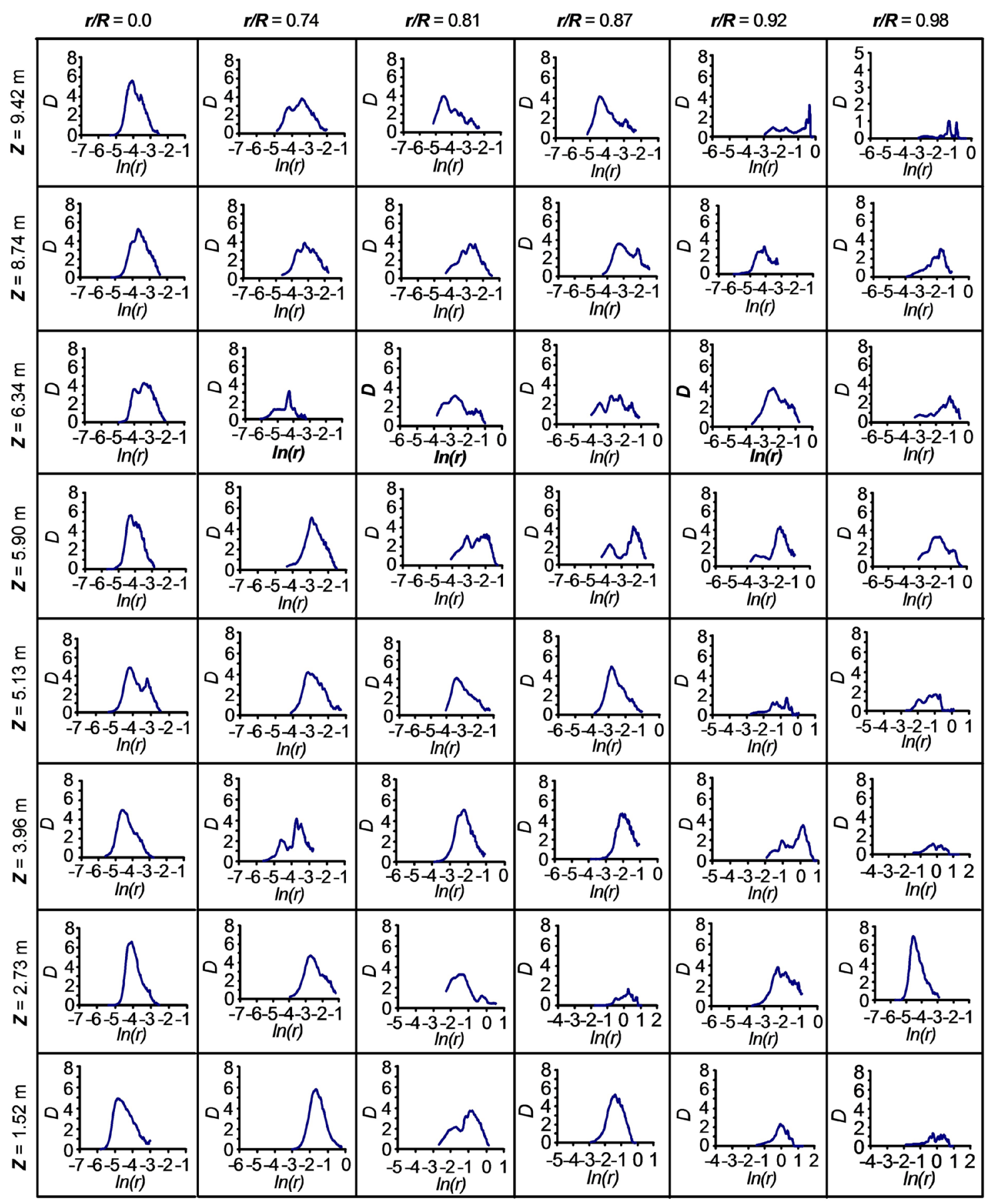

Figure 6. Mapping of correlation dimension along $\ln (r)$ axis for solid concentration signals from the wall-region of the high-flux riser at $U_{g}=10 \mathrm{~m} / \mathrm{s}, G_{s}=300 \mathrm{~kg} / \mathrm{m}^{2} \mathrm{~s}$. 
Contrary to the wall region where there is low degree of freedom for particles, there is less interactions due to high solid concentration, presence of solid clusters and the boundary layer or wall effects, all of which limits particle's motions in the gas-solid suspensions leading to low values of the correlation dimension. Studies show that the dilute phase and the dense or cluster phase have different dynamical properties and therefore shows different dynamic behaviors [3] which are described by the differences shown by correlation dimension profiles. The dilute phase shows more complex time variations of solid concentrations while the dense or cluster phase with high solid concentrations is less complex and hence lower peak for correlation dimension profiles. Further it is shown that both dilute and cluster phases are strongly coupled system at the wall of the riser with the dilute phase dominating [3].

Lower peak values correlation dimension are generally found for conditions with high solids concentration, due to the fact that the presence of high solids concentration in the gas phase near the wall reduces the amount of turbulence and complexity of flow [4] [6].

Towards the wall both phases are partially coupled system with cluster or dense phase dominating leading to low correlation dimension [3]. At high solid concentration there is an increased formation of uniform or homogenized clusters which increase in size with time leading to the decreased solid concentration fluctuations and clusters mobility. This in turn leads to the lowered degree of freedom of particles between the clusters and in the dilute phase.

Results show a tendency of change in location of the peak value, i.e., the maximum value of $D_{3}$ along the $\ln (r)$ axis (horizontal position). The maximum $D_{3}$ shifts from left to right along the $\ln (r)$ axis towards the origin of the $D_{3}$ versus $\ln (r)$ plane as one move towards the wall. Also for all elevations, towards the wall of the riser reactor (i.e., $0.74 \leq r / R \leq 0.98$ ) correlation dimension plots show the presence of the multi-fractal behavior which manifests by the presence of more than one local maximum value indicating two or more peak values of $D_{3}$. Presence of two local maximum values indicates the bifractal behavior of the gas-solid flow. Such behaviors were observed and become more dominant towards the wall when the gas velocity, $U_{g}=10 \mathrm{~m} / \mathrm{s}$ and solid flux, $G_{s},=300 \mathrm{~kg} / \mathrm{m}^{2} \mathrm{~s}$, as shown in Figure 6. This observation is found in high density gas-solids flow behavior, where dilute-phase and dense-phases co-exist. As shown in Figure 4 at $U_{g}=8 \mathrm{~m} / \mathrm{s}$ and solid flux, $G_{s}=300 \mathrm{~kg} / \mathrm{m}^{2} \mathrm{~s}$ this behavior is found in the bottom section at $Z=1.52 \mathrm{~m}$ and $r / R=0.0, \mathrm{Z}=2.73 \mathrm{~m}$ and $0.74 \leq \mathrm{r} / \mathrm{R} \leq 0.87$; in the flow developing section at $\mathrm{Z}=3.96 \mathrm{~m}$ and $0.81 \leq r / R \leq 0.98$ and also in the top section of the riser at $\mathrm{Z}=9.42 \mathrm{~m}, 0.74 \leq r / R \leq 0.98$.

According to Figure 5, this behavior is also found in the bottom section of the riser $Z=1.52 \mathrm{~m}$ and $r / R=0.81, Z=2.73$ and $0.87 \leq r / R \leq 0.92$. But also in the developed-flow section at $Z=6.34 \mathrm{~m}$ very close to the wall $(0.92 \leq r / R \leq 0.98)$. When the operating conditions are changed to $U_{g}=10 \mathrm{~m} / \mathrm{s}, G_{s}=300 \mathrm{~kg} / \mathrm{m}^{2} \mathrm{~s}$ (which is presented by Figure 6) this multifractal behaviour emerges in the bot- 
tom section at $Z=1.52 \mathrm{~m}$ with $r / R=0.81$ and $0.98, \mathrm{Z}=3.96 \mathrm{~m}$ and $0.92 \leq r / R \leq$ 0.98 , and in the mid-height of the riser at $Z=5.90 \mathrm{~m}$ between $0.81 \leq r / R \leq 0.98$.

The single peak values of the correlation dimension profiles at the centre in all axial levels indicates the uniform flow structure or low tendency to the formation of segregated flow. The multiple peaks of correlation dimension profiles with varying magnitudes towards the wall indicates the presence of multifractal flow structures which can be attributed to the presence of dense phases dominated by particle aggregates with varying solid concentrations leading to the mixed flow behaviors, as stated above.

\subsection{Analysis of the Shapes of Correlation Dimension Profiles from the Wall Region}

The analysis of the shapes of correlation dimension profiles reveals three different types, i.e., A, B and $\mathrm{C}$ as shown in Figure 7. Type A correlation dimension profiles have a single tall peak which are mostly found at the centre $(r / R=0.0)$ for all operating conditions especially in the fully developed section $(Z=9.42 \mathrm{~m})$. They are also observed in some parts of the wall region in the flow-development section, $Z=3.96 \mathrm{~m}$. This type of profiles indicates a uniform and low solid concentration flow structure.

Type B profiles are double peaked profiles, which emerge for all operating conditions in various spatial locations of the riser. They are mostly found in the wall region, for instance, in the entrance section, $Z=1.52 \mathrm{~m}$, close to the wall for which $r / R=0.92$ and at $r / R=0.74, Z=2.73 \mathrm{~m}$. They are also found in the flow development section at $r / R=0.81$ and 0.98 for $Z=3.96 \mathrm{~m}$ and $Z=3.16 \mathrm{~m}$ for $U_{\mathrm{g}}$ $=5.5 \mathrm{~m} / \mathrm{s}$ and $G_{\mathrm{s}}=300 \mathrm{~kg} / \mathrm{m}^{2} \mathrm{~s}$. At $U_{\mathrm{g}}=8 \mathrm{~m} / \mathrm{s}$ and $G_{\mathrm{s}}=300 \mathrm{~kg} / \mathrm{m}^{2} \mathrm{~s}$ such profiles are observed in the flow development section at $r / R=0.87$ and $Z=3.16 \mathrm{~m}$. When $U_{\mathrm{g}}$ was increased to $10 \mathrm{~m} / \mathrm{s}$ at $G_{\mathrm{s}}=300 \mathrm{~kg} / \mathrm{m}^{2} \mathrm{~s}$, the double peaked correlation dimension profiles appeared, for instance, in the entrance section at $r / R=$ 0.81 for $Z=1.52$ and $2.73 \mathrm{~m}$, in the developed flow section at $r / R=0.81$ and 0.87 for $Z=5.90 \mathrm{~m}$ and in the fully developed flow section $r / R=0.87$ and $Z=8.74 \mathrm{~m}$. Type B correlation dimension profiles indicate two dominant flow types in the wall region of the riser, attributable to relatively dilute phase and dense or cluster phase.

Type C correlation dimension profiles are multiple peaked profiles. They are found in some locations of the wall region for all operating conditions and in all sections of the riser. For instance, they are found in the entrance section at $r / R=$ 0.87 and $Z=2.73 \mathrm{~m}$; in the flow developing section at $r / R=0.87$ and $Z=3.96 \mathrm{~m}$; and in the fully developed flow section towards the riser exit at $r / R=0.87$ and $Z$ $=8.74 \mathrm{~m}$ and $r / R=0.74$ and 0.87 for $Z=9.42 \mathrm{~m}$ for $U_{\mathrm{g}}=5.5 \mathrm{~m} / \mathrm{s}$ and $G_{\mathrm{s}}=300$ $\mathrm{kg} / \mathrm{m}^{2} \mathrm{~s}$. For $U_{\mathrm{g}}=8 \mathrm{~m} / \mathrm{s}$ and $G_{\mathrm{s}}=300 \mathrm{~kg} / \mathrm{m}^{2} \mathrm{~s}$ these profiles were observed in the entrance section at $r / R=0.87$ and 0.92 for $Z=2.73 \mathrm{~m}$. When $U_{\mathrm{g}}$ was increased to $10 \mathrm{~m} / \mathrm{s}$ at $G_{\mathrm{s}}=300 \mathrm{~kg} / \mathrm{m}^{2} \mathrm{~s}$ these profiles were observed in the entrance section for instance at $r / R=0.81$ and $Z=1.52 \mathrm{~m}$; in the developing flow section at $r / R=$ 
0.74 and $Z=3.96 \mathrm{~m}$; in the transition at $r / R=0.87$ and $Z=6.34 \mathrm{~m}$ and in the fully developed section towards the exit section they are found at $r / R=0.81$ and $Z=9.42 \mathrm{~m}$. Multiple peak correlation dimension profiles indicate more than two dominant flow patterns, attributable to dilute phase, clusters and intensive interactions between these two phases with the wall.

\subsection{Mapping of Correlation Dimension, $D_{3}$, on the $D_{3}-\ln (r)$ Plane}

Analysis of mapped correlation dimension profiles was used to investigate the change of gas-solid flow dynamics in the riser by observing shapes of the profiles, their positions on the $\ln (r)$ axis and the maximum value of $D_{3}$. Mapping of correlation dimension, $D_{3}$, on the $D_{3}-\ln (r)$ plane is shown in Figure 8 for different $U_{\mathrm{g}}$ at three axial elevations. This approach provides a new method of studying the flow dynamics in a high-flux riser.

Figure 8 shows also the mapping of the $D_{3}$ profiles along the $\ln (r)$ axis at $U_{g}=$ $5.5,8$ and $10 \mathrm{~m} / \mathrm{s}$ for $G_{\mathrm{s}}=300 \mathrm{~kg} / \mathrm{m}^{2} \mathrm{~s}$ in the entrance, developing flow and developed flow sections, that is, $\mathrm{Z}=1.52,3.96$ and $9.42 \mathrm{~m}$ respectively. It was further observed that the $D_{3}$ profiles at the center $(r / R=0.0)$ forms a higher peak which relatively increases towards the top of the riser in the fully developed flow section. The rest of the profiles in the wall regions are flatter at the top of the riser, compared to those in the flow developing and entrance sections. That is, in the entrance section the profiles close to the wall $(r / R=0.98)$ have higher peaks or higher correlation dimension than the profiles at the top of the riser. This is attributable to intensive mixing in the entrance section after the gas passes the distributor plate.

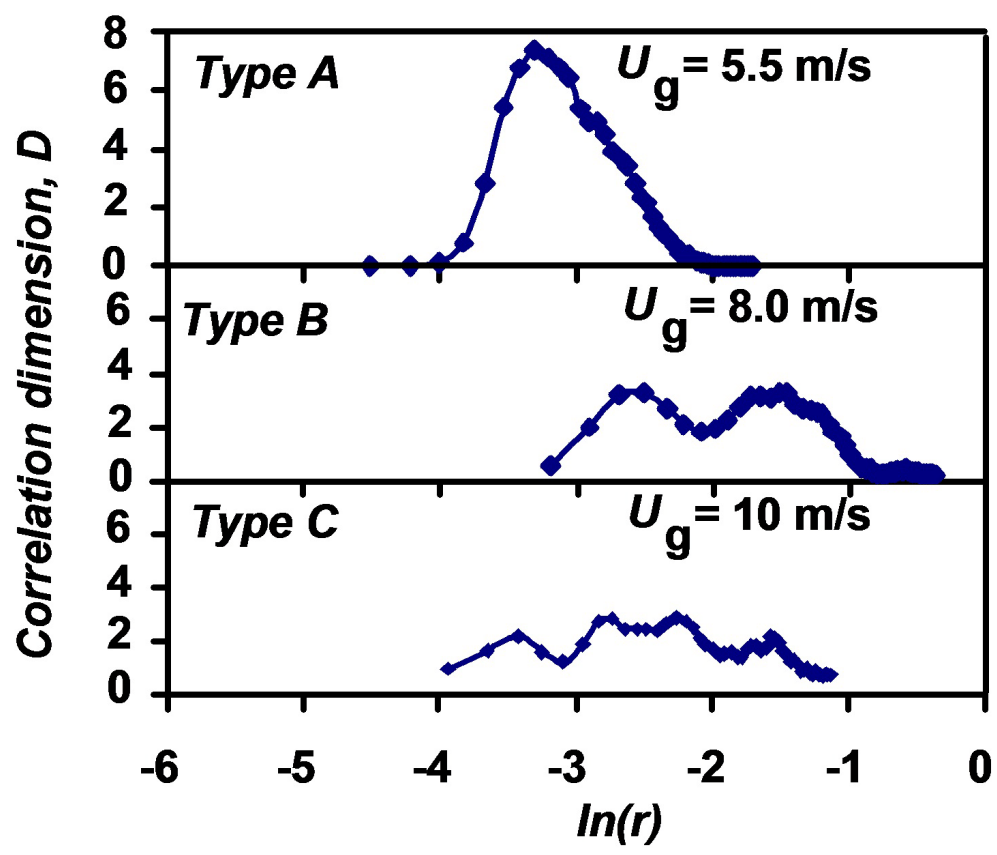

Figure 7. Characterizing correlation dimension profiles obtained at different operating conditions. 


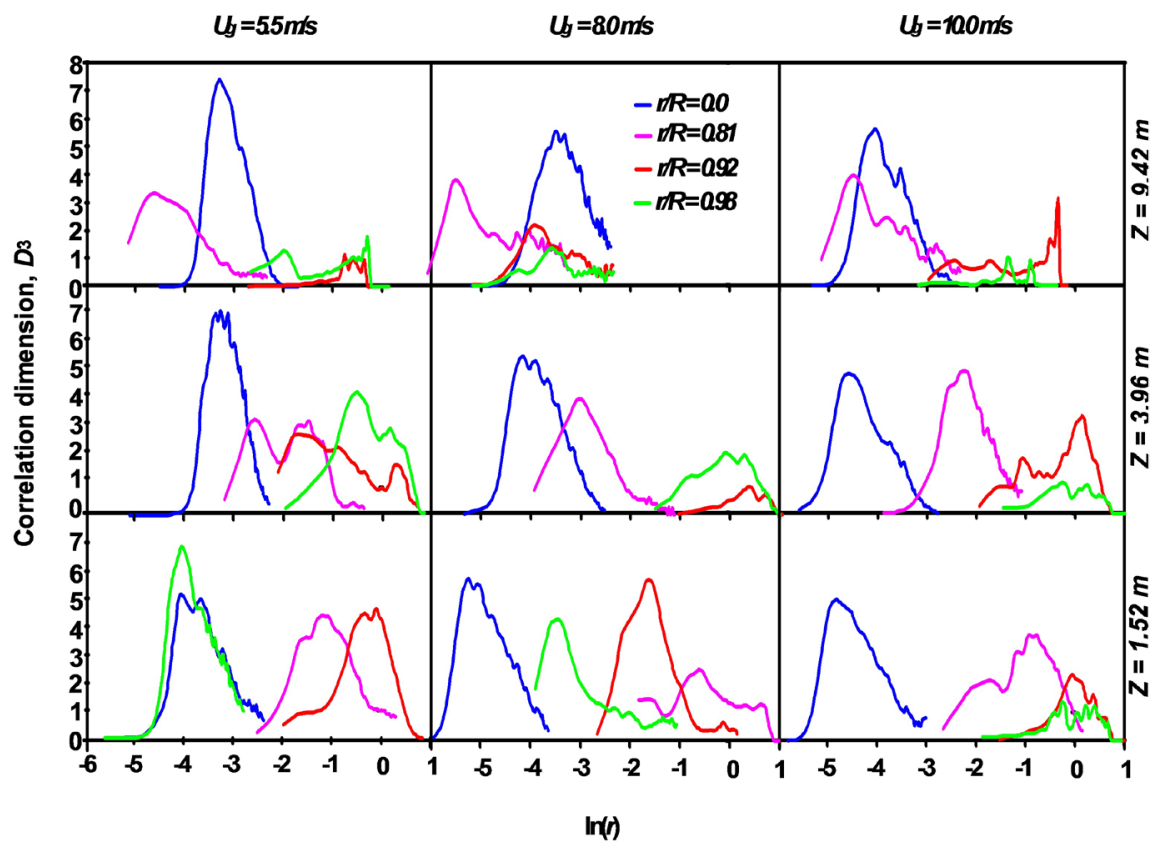

Figure 8. Mapping of $D_{3}$ on $D_{3}-\ln (\mathrm{r})$ plane in the wall region and different flow development sections for different $U_{\mathrm{g}}$ at $G_{\mathrm{s}}=300 \mathrm{~kg} / \mathrm{m}^{2}$ s.

The position of the correlation dimension profiles at $r / R=0.0$ along the $\ln (r)$ axis is relatively the same for all sections. However, the profiles in the wall region have the tendency to shift their positions along the $\ln (r)$ axis depending on gas velocity, $U_{\mathrm{g}}$, and axial elevation, $Z$. This indicates that the scaling regions of the reconstructed attractors change their position and size. When the profiles shift to the right, it implies that attractors expand in size and the distances between trajectories also increases. It can be observed that the profile at $r / R=0.81$ changes position towards the negative direction along the $\ln (r)$ axis from the entrance $(0.5$ to -2.5$)$, developing $(-0.5$ to -3.2$)$ and the developed $(-2.5$ to -5.2$)$ sections at the top of the riser. The profiles also show a clear double peak in the flow development section $(Z=3.96 \mathrm{~m})$. The profile close to the wall $(r / R=0.98)$ in the entrance section $(Z=1.52 \mathrm{~m})$ is located in the same position (around -2.5 to -5.5 ) with the centre profile with its peak being the highest. This suggests resemblance in the gas-solid flow behavior. The peak of this profiles decrease along the high flux riser from the entrance section through the developing up to the developed section. This behavior is also shown by the profiles at $r / R=0.92$.

At $U_{g}=8 \mathrm{~m} / \mathrm{s}$, the centre profile (at $r / R=0.0$ ) shows a small shift towards the right side or positive direction along the $\ln (r)$ axis from the entrance section where it is located around -3.7 to -6.5 towards the fully developed flow section around -2.5 to -4.9 . The correlation dimension profiles at the wall show the tendency to shift towards the negative direction along the $\ln (r)$ axis as $Z$ increases as observed also at $U_{\mathrm{g}}=8 \mathrm{~m} / \mathrm{s}$. In the entrance section, the profile at $r / R=$ 0.81 is located from 0 to -3 and shifts to a location between -1 and -4 in the developing flow section and then from -3.4 to -6 in the fully developed flow 
section. The profiles close to the wall, at $r / R=0.92$ and 0.98 , are located in the same span in the flow developing section $(1$ to -1.5$)$ and fully-developed flow section $(-2.4$ to -5.1$)$ while in the entrance section the profile at $r / R=0.98$ is located on the left $(-1$ to -4$)$ of the profile at $r / R=0.92$ (that is from 0.2 to -2.8 ). Also these profiles have low peaks compared to the other profiles.

Further Figure 8 shows also mapping of the $D_{3}$ profiles at $U_{\mathrm{g}}=10 \mathrm{~m} / \mathrm{s}$ and $300 \mathrm{~kg} / \mathrm{m}^{2} \mathrm{~s}$ in the three flow development sections, that is, entrance $(Z=1.52$ $\mathrm{m})$, flow development $(Z=3.96 \mathrm{~m})$ and fully developed $(Z=9.42 \mathrm{~m})$ sections. Figure 8 shows that at this operating condition again the centre profile lies in a relatively same location spanning from around -2.6 to -5.8 in all sections. However, the profile at $r / R=0.81$ shows to shift towards the left from the entrance (around 0.2 to -2.7$)$, developing ( -1 to -4$)$ to the developed $(-2.5$ to -5.2$)$ sections along the $\ln (r)$ axis.

The tendency of $D_{3}$ profiles to form multiple peaks closer to the wall at $r / R=$ 0.92 and 0.98 , increases especially in the entrance section at $U_{\mathrm{g}}=10 \mathrm{~m} / \mathrm{s} \mathrm{com}$ pared to the center profiles. These observations can be associated to the increasing solid concentration and formation of dense phases which reduces the degree of freedom of particles. Also the influence of the wall on the gas phase and solid particles becomes significant. All these reduce particles momentum and interactions with gas phase and the wall resulting into less complex flow behavior and hence low values of correlation dimensions towards the wall. Multiple peaks with lower varying magnitudes of correlation dimensions towards the wall are attributed to the presence of low dimensional multifractal flow behavior which results from the presence of dense phases which are dominated by clusters or particle aggregates with varying solid concentrations which leads to the mixed phases flow behaviors.

The span of the $D_{3}$ profiles from left to right indicate changes in the size of the reconstructed attractor in phase space. For example, in Figure 8, for $U_{g}=5.5$ $\mathrm{m} / \mathrm{s}$, at $r / R=0.92$ and $Z=9.42 \mathrm{~m}$, the profile spans within -1 and 0 , while at $Z=$ $1.52 \mathrm{~m}$, the profile span from -2 to 1 along the $\ln (r)$ axis. A profile of $D_{3}$ on the right hand side indicates that the scaling region(s) of the attractor is within longer trajectory distances, implying expanded attractor. The radial position $r / R$ $=0.81$ exhibits a wide range of the changes in the flow dynamics in the gas-solids flow along the axial elevations of the riser. This has been explained in literature using core-annulus model, which was based on changes in radial solids concentration and particle velocities [23]. It is found that the maximum values of $D_{3}$ vary for the profiles from different locations and at different operating conditions. Also there are wide variations in the location of the profiles along $\ln (r)$ axis from 0 to -5.5 which forms on other property of gas-solid flow dynamics developed in this study. This indicates that the microscopic flow scales (originally captured by single solids concentration signal) diminishes from largest at the entrance to the smallest at the top of the riser. That is, at $r / R=0.81$, changes occur from densest to the most dilute flow behavior along the riser. 


\subsection{Maximum Correlation Dimension Profiles in the Wall Region of the High Flux Riser}

Variation of the maximum correlation dimension, $D_{\max }$, determined from the correlation dimension profiles of $D_{3}$, along the radial position at a given axial elevations and operating conditions are shown in Figure 9. Closer to the wall, at $r / R=0.98$ the values of $D_{\max }$ along the axial direction are relatively higher at $U_{g}$ $=5.5 \mathrm{~m} / \mathrm{s}$ and $G_{s}=300 \mathrm{~kg} / \mathrm{m}^{2} \mathrm{~s}$ except at at $Z=2.73 \mathrm{~m}$ and $8.74 \mathrm{~m}$. Lower values were observed in the bottom section $(Z=1.52 \mathrm{~m})$, transition $(Z=3.96 \mathrm{~m})$ and top $(Z=9.42)$ sections. Also away from the wall towards the centre, $D_{\max }$ profiles are irregular revealing the non-uniform time-variations in the flow behavior of the gas-solid flow depicted by solids concentrations signals in the riser reactor. For $r / R=0.81$, again, using normal radial profiles of $D_{\max }$ one cannot notice the inherent dynamical changes along the riser in this radial position. Thus, the current study used $D_{3}$ profiles to show that this radial position exhibit strongest variations in the gas-solids flow dynamics. Using the values of $D_{\max }$ could not reveal this phenomenon (Figure 9) compared to details revealed using profiles in Figure 8.

\subsection{Effects of Gas Velocity on Correlation Dimension $\left(D_{3}\right)$}

The effects of changing gas velocity on the correlation dimension and its profiles were studied. Figure 10 shows the effects of gas velocity $\left(U_{\mathrm{g}}\right)$ on the Correlation Dimension $\left(D_{3}\right)$ at different radial position $(r / R)$ in selected sections of the riser, using profiles generated from local solid concentration.

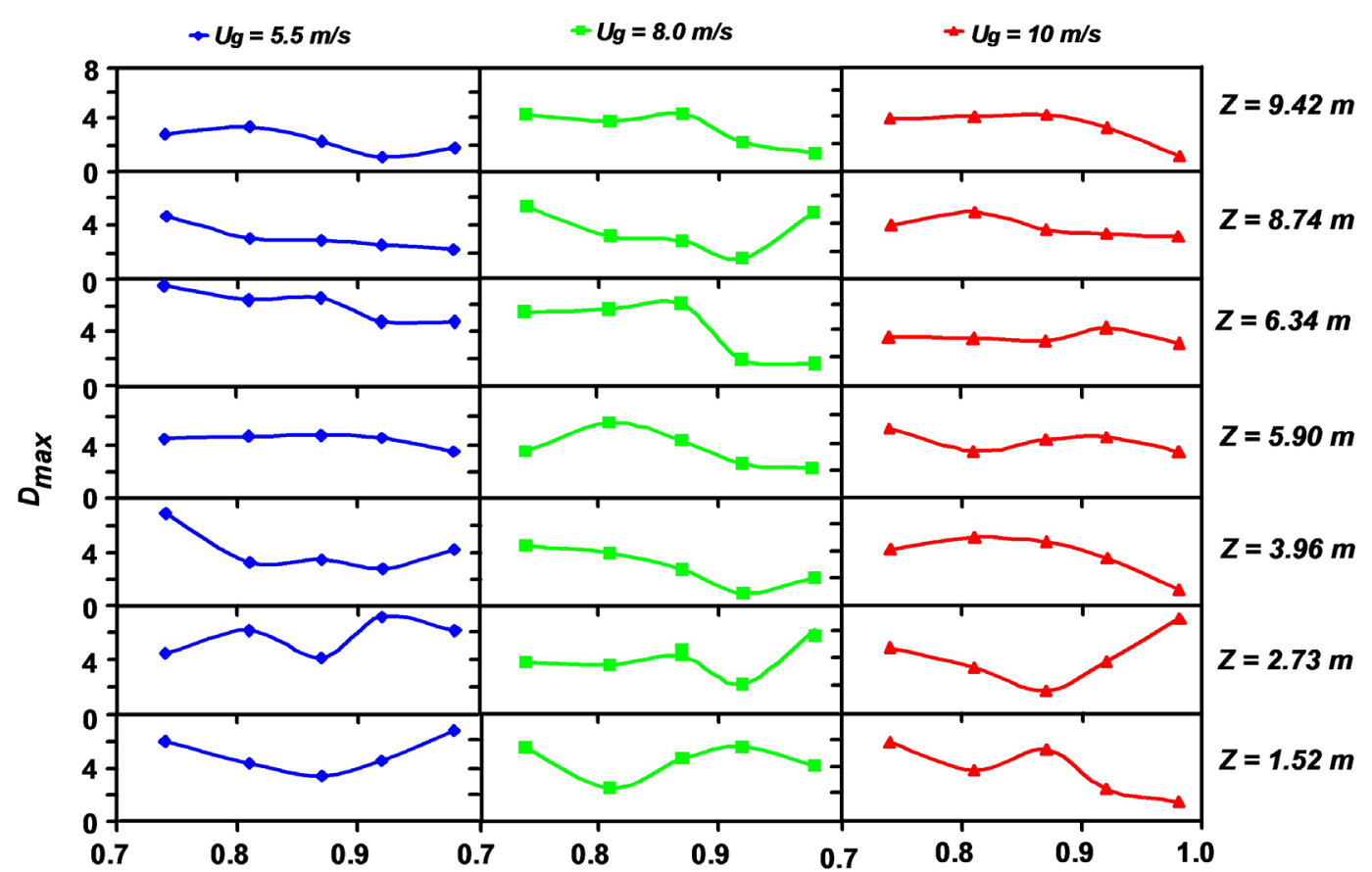

Figure 9. Radial profiles of maximum correlation dimension versus radial positions plots at $G_{\mathrm{s}}=300$ $\mathrm{kg} / \mathrm{m}^{2} \mathrm{~s}$. 


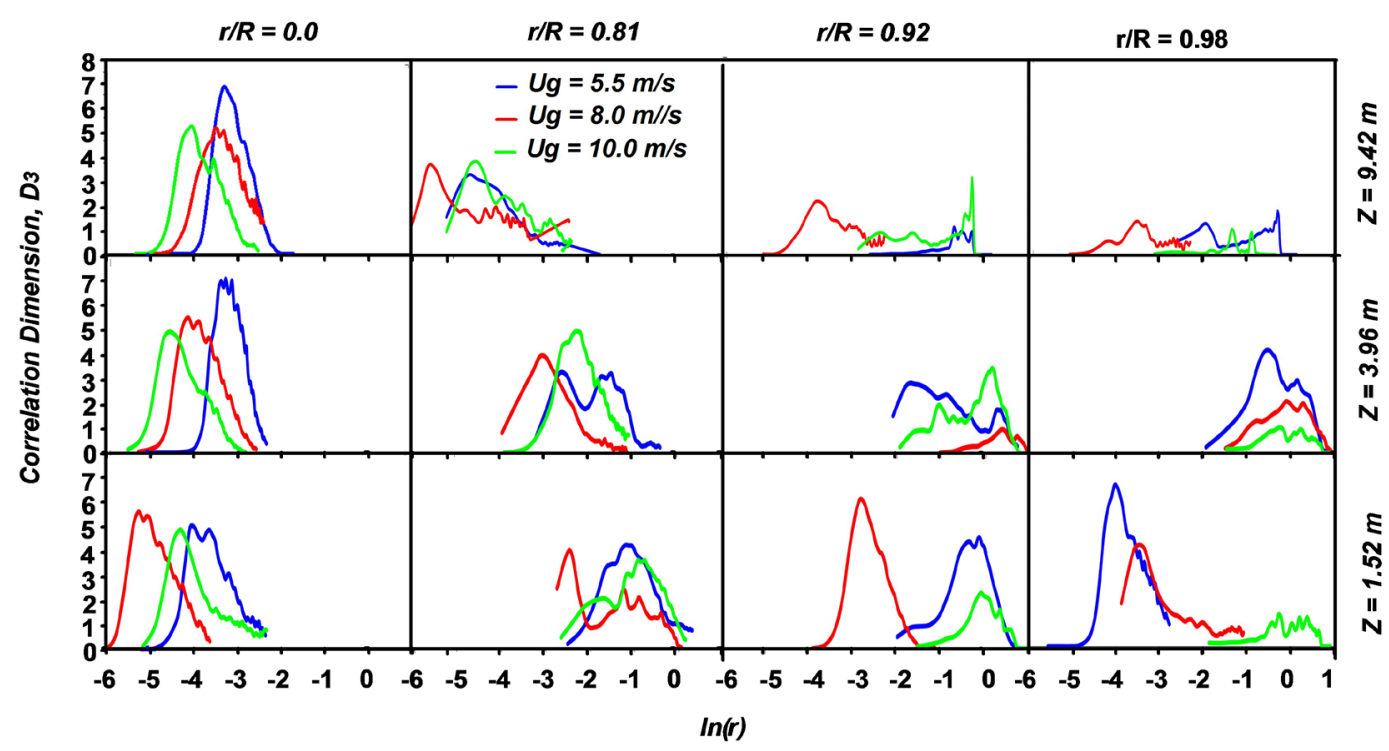

Figure 10. Effects of gas velocity $\left(U_{\mathrm{g}}\right)$ on correlation dimension $\left(D_{3}\right)$ at different radial position $(r / R)$ along the risers' flow development sections at $G_{s}=300 \mathrm{~kg} / \mathrm{m}^{2} \mathrm{~s}$.

Figure 10 shows the correlation dimension profiles in the developed flow section of the riser $(Z=9.42 \mathrm{~m})$. For all gas velocities, the profiles at centre $(r / R=$ 0.0 ) have higher peak compared to those from other locations. Also the profiles show a shift towards lower hyperspherical radius as the velocity increases. In the wall region profiles are flatter with multiple peaks and have no clear trend or pattern. However, at high velocity, $U_{\mathrm{g}}=8$ and $10 \mathrm{~m} / \mathrm{s}$, the correlation dimension profiles are located within a higher hyperspherical radius of the phase space especially at $r / R=0.92$ and 0.98 .

In the flow development section, $Z=3.96 \mathrm{~m}$, (as per Figure 10), the profile at the centre $(r / R=0.0)$ and at lower velocity $(5.5 \mathrm{~m} / \mathrm{s})$ have higher peak compared to other profiles. A closer look of the profiles at various radial positions $(r / R=$ $0.81,0.92$ and 0.98 ) shows a clear shift of the profiles towards the origin of the plane, i.e. all profiles at all velocities investigated, i.e., $U_{\mathrm{g}}=5.5,8$ and $10 \mathrm{~m} / \mathrm{s}$ are located in the higher hyperspherical radius along $\ln (r)$ axis, which signifies expanded attractors with longer trajectory distances near the wall.

As shown in Figure 10 the profiles in the entrance section $(Z=1.52 \mathrm{~m})$ at the centre and in the wall region does not show a clear trend or pattern. However, at high velocity, i.e., $U_{\mathrm{g}}=10 \mathrm{~m} / \mathrm{s}$, the correlation dimension $\left(D_{3}\right)$ profiles are located within a higher hyperspherical radius of the phase space especially at $r / R=$ $0.81,0.92$ and 0.98 . Such observation of changes in the dynamics have not been reported using average values of solids concentration signals or single value chaos invariants in the past, when $r / R, U_{g}$ and $Z$ were changed. This study focused on the dynamics of gas-solid flow in the wall region of the high-flux gas-solid riser while other studies considered the whole cross-section of the risers. The shift of maximum correlation dimension or scaling region along the $\ln (r)$ axis is being reported in this study for the first time. 
The shifting tendency of the correlation dimension profiles is further summarized in Table 1 which presents how the change in velocities affects the position at which the maximum $D_{3}$ is located along the $\ln (r)$ axis. The analysis is presented for different radial positions (the centre and the wall region) along the riser height from the entrance section $(Z=1.52 \mathrm{~m})$, flow development section $(Z$ $=3.96 \mathrm{~m})$ and in the fully developed flow section $(Z=9.42 \mathrm{~m})$. For instance, in the entrance section $(Z=1.52 \mathrm{~m})$ close to the wall $(r / R=0.98)$, when $U_{g}=5.5$ $\mathrm{m} / \mathrm{s}$ the maximum D3 is located at $\ln (r)=-4.05$, while for $U_{g}=8.0 \mathrm{~m} / \mathrm{s}$ it is located at $\ln (r)=-3.38$ and for $U_{g}=10 \mathrm{~m} / \mathrm{s}$ it is located at $\ln (r)=-0.26$.

\subsection{Effects of Riser Height $(Z)$ on Correlation Dimension $\left(D_{3}\right)$}

The effect of the axial elevations was investigated in three sections of the riser, i.e., the entrance, developing and the fully developed sections. Figure 11 shows the effects of riser height $(Z)$ on correlation dimension $\left(D_{3}\right)$ at different radial position $(r / R)$ for the selected operating conditions.

In Figure 11 correlation dimension $\left(D_{3}\right)$ profiles at different axial elevation (Z)at $U_{\mathrm{g}}=5.5 \mathrm{~m} / \mathrm{s}$ and $G_{\mathrm{s}}=300 \mathrm{~kg} / \mathrm{m}^{2} \mathrm{~s}$ are shown. The peak values of the correlation dimension in the fully developed section $(Z=9.42 \mathrm{~m})$ are lower compared to other elevations at the wall. Also at $r / R=0.81$ as the height increases from $Z=1.52 \mathrm{~m}$ to $9.42 \mathrm{~m}$ the profiles shifts along the horizontal axis towards the lower hyperspherical radius. Close to the wall at $r / R=0.98$ the correlation dimension profiles in the entrance section $(Z=1.52 \mathrm{~m})$ has higher peak values at $U_{\mathrm{g}}=5.5$ and $8 \mathrm{~m} / \mathrm{s}$ but are located at the lowest value of the hyperspherical radius which indicates contraction of attractor which implies higher solid concentration.

Correlation dimension profiles at different axial elevation ( $Z$ ) for $U_{\mathrm{g}}=8 \mathrm{~m} / \mathrm{s}$ and $G_{\mathrm{s}}=300 \mathrm{~kg} / \mathrm{m}^{2} \mathrm{~s}$ are also shown in Figure 11 . At the centre of the riser $(r / R$ $=0.0)$ the correlation dimension profiles are similar for different elevations along the riser, that is, in the entrance sections $(Z=1.52 \mathrm{~m})$, flow developing section $(Z=3.96 \mathrm{~m})$ and in the fully developed section $(Z=9.42 \mathrm{~m})$. This indicates high uniformity of the flow structure at the centre. However, as the height increases, the location of the correlation dimension profiles shifts to a higher hyperspherical radius. In the wall region at $r / R=0.81$ the trend is vice versa. The profile at $Z=1.52 \mathrm{~m}$ is located at higher hyperspherical radius and as the height increases the location of the profiles shifts to the lower hyperspherical radius. Close to the wall at $r / R=0.92$ and 0.98 , profiles have similar trend where the profiles at $Z=3.96 \mathrm{~m}$ are located at the higher hyperspherical radius and the profile at $Z=9.42 \mathrm{~m}$ locating at the lowest value.

Further, the correlation dimension profiles at different axial elevation $(Z)$ for $U_{\mathrm{g}}=10 \mathrm{~m} / \mathrm{s}$ and $G_{\mathrm{s}}=300 \mathrm{~kg} / \mathrm{m}^{2} \mathrm{~s}$ is also shown in Figure 11 . The trend of the profile at this operating condition is similar to the trend at $U_{\mathrm{g}}=8 \mathrm{~m} / \mathrm{s}$ and $G_{\mathrm{s}}=$ $300 \mathrm{~kg} / \mathrm{m}^{2}$ s except at $r / R=0.92$ and 0.98 . The correlation dimension profiles at the centre of the riser $(r / R=0.0)$ in the entrance sections $(Z=1.52 \mathrm{~m})$, flow 
Table 1. Location of the maximum $D_{3}$ along $\ln (r)$ axis at the centre and wall region at $U_{\mathrm{g}}$ $=5.5,8$ and $10 \mathrm{~m} / \mathrm{s}$ in the entrance, flow-development and developed-flow sections of the riser.

\begin{tabular}{|c|c|c|c|c|}
\hline$Z(m)$ & $r / R$ & $U_{\mathrm{g}}(\mathrm{m} / \mathrm{s})$ & $\begin{array}{c}\text { Value of maximum } \\
D_{3}\end{array}$ & $\begin{array}{c}\text { Location of } \\
\text { maximum } D_{3} \text { along } \ln (r)\end{array}$ \\
\hline \multirow{12}{*}{9.42} & \multirow{3}{*}{0.0} & 5.5 & 7.36 & -3.31 \\
\hline & & 8 & 5.50 & -3.47 \\
\hline & & 10 & 5.60 & -4.04 \\
\hline & \multirow{3}{*}{0.81} & 5.5 & 3.35 & -4.64 \\
\hline & & 8 & 3.73 & -5.56 \\
\hline & & 10 & 3.90 & -4.46 \\
\hline & \multirow{3}{*}{0.92} & 5.5 & 1.11 & -0.77 \\
\hline & & 8 & 2.15 & -3.86 \\
\hline & & 10 & 3.13 & -0.35 \\
\hline & \multirow{3}{*}{0.98} & 5.5 & 1.77 & -0.30 \\
\hline & & 8 & 1.36 & -3.55 \\
\hline & & 10 & 1.00 & -1.38 \\
\hline \multirow{12}{*}{3.96} & \multirow{3}{*}{0.0} & 5.5 & 7.09 & -3.26 \\
\hline & & 8 & 5.50 & -4.14 \\
\hline & & 10 & 4.93 & -4.61 \\
\hline & \multirow{3}{*}{0.81} & 5.5 & 3.25 & -1.46 \\
\hline & & 8 & 3.97 & -3.08 \\
\hline & & 10 & 5.00 & -2.28 \\
\hline & \multirow{3}{*}{0.92} & 5.5 & 2.79 & -1.58 \\
\hline & & 8 & 0.97 & 0.36 \\
\hline & & 10 & 3.43 & 0.14 \\
\hline & \multirow{3}{*}{0.98} & 5.5 & 4.26 & -0.56 \\
\hline & & 8 & 2.14 & -0.11 \\
\hline & & 10 & 1.07 & -0.26 \\
\hline \multirow{12}{*}{1.52} & \multirow{3}{*}{0.0} & 5.5 & 5.05 & -3.98 \\
\hline & & 8 & 5.68 & -5.24 \\
\hline & & 10 & 4.94 & -4.31 \\
\hline & \multirow{3}{*}{0.81} & 5.5 & 4.35 & -1.21 \\
\hline & & 8 & 4.13 & -2.46 \\
\hline & & 10 & 3.72 & -0.93 \\
\hline & \multirow{3}{*}{0.92} & 5.5 & 4.58 & -0.08 \\
\hline & & 8 & 6.16 & -2.83 \\
\hline & & 10 & 2.31 & -0.05 \\
\hline & \multirow{3}{*}{0.98} & 5.5 & 6.80 & -4.05 \\
\hline & & 8 & 4.20 & -3.38 \\
\hline & & 10 & 1.35 & -0.26 \\
\hline
\end{tabular}




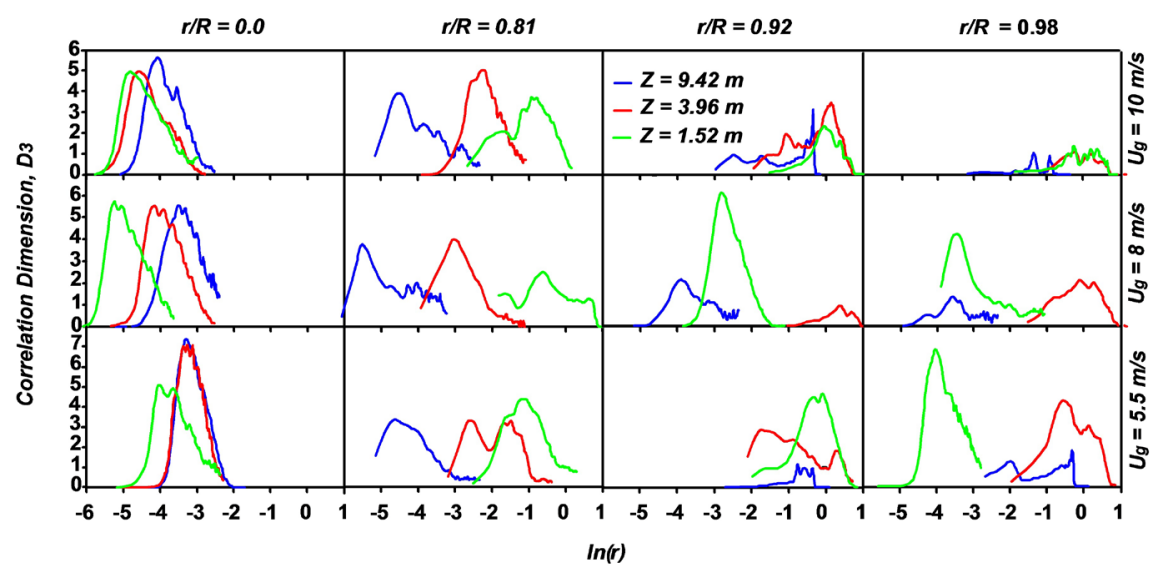

Figure 11. Effects of riser height $(\mathrm{Z})$ on correlation dimension $\left(\mathrm{D}_{3}\right)$ at different radial positions $(\mathrm{r} / \mathrm{R})$ and gas velocities $\left(\mathrm{U}_{\mathrm{g}}\right)$ at $\mathrm{G}_{\mathrm{s}}=300 \mathrm{~kg} / \mathrm{m}^{2}$ s.

developing section $(Z=3.96 \mathrm{~m})$ and in the fully developed section $(Z=9.42 \mathrm{~m})$ shifts towards the higher hyperspherical radius as the height increases. Also in the wall region at $r / R=0.81$ the trend is vice versa. The profile at $Z=1.52 \mathrm{~m}$ is located at higher hyperspherical radius and as the height increases the location of the profiles shifts to the lower hyperspherical radius. Profiles at $r / R=0.92$ and 0.98 for all axial elevations studied are located in the same locations of the hyperspherical radius with higher peak value at $r / R=0.92$ than at $r / R=0.98$.

The effect of riser height can be observed further through the shifting tendency of the correlation dimension profiles as shown also in Table 1. The change in riser height affects the positions at which the maximum $D_{3}$ is located along the $\ln (r)$ axis in the centre and the wall region for all axial elevations of the riser. For instance, close to the wall $(r / R=0.98)$ when $U_{\mathrm{g}}=5.5 \mathrm{~m} / \mathrm{s}$, in the entrance section, $Z=1.52 \mathrm{~m}$, the maximum $D_{3}$ is located at $\ln (r)=-4.05$, while in the flow developing section, $Z=3.96 \mathrm{~m}$, it is located at $\ln (r)=-0.56$ and in the fully developed section, $Z=9.42 \mathrm{~m}$, it is located at $\ln (r)=-0.30$.

\section{Conclusion}

Gas-solid flow dynamics in the high-flux CFB riser was studied by mapping the correlation dimension, $D_{3}$, at different spatial locations for different operating conditions $\left(U_{\mathrm{g}}\right)$ and locations along the $\ln (r)$ axis. The number of peaks for each profile was also assessed. Based on the discussion, it can be concluded that the three-step correlation dimension $\left(D_{3}\right)$ profiles are good indicator of the differences in the flow dynamics over various states of the gas-solid flow in the riser. These correlation dimension profiles at the centre are mostly single peaked with higher peak values than the wall region showing uniform flow dynamics. These peak values decrease towards the wall. Towards the wall these profiles exhibit double or multiple peaks profiles showing bifractal or multifractal flow behavior. As the velocity increases the correlation dimension profiles close to the wall become more random and irregular affirming chaotic nature in these locations. Further, towards the wall these profiles change location towards higher hyper- 
spherical radius of the attractor at $Z=9.42 \mathrm{~m}$. As the height increases the correlation dimension plot shifts towards higher hyperspherical radius at the centre and towards lower hyperspherical radius in the wall at $r / R=0.81$. Mapping of the established correlation dimension $\left(D_{3}\right)$ profiles forms a good tool of analysis of the flow dynamics in the high-flux gas-solid riser compared to other tools of analysis. The study recommends further analysis to other risers of different dimensions operated at high-flux conditions using the established method.

\section{Conflicts of Interest}

The authors declare no conflicts of interest regarding the publication of this paper.

\section{References}

[1] Johnsson, J., Zijerveld, R.C., Schouten, J.C., van den Bleek, C.M. and Leckner, B. (2000) Characterization of Fluidization Regimes by Time-Series Analysis of Pressure Fluctuations. International Journal of Multiphase Flow, 26, 663-715. https://doi.org/10.1016/S0301-9322(99)00028-2

[2] Ahuja, P., Agrawal, H., Sethi, A.K. and Raj, U. (2005) Chaotic Analysis of Pressure Fluctuations in a Gas-Solid Fluidized Bed. Indian Journal of Chemical Technology, $12,212-219$

[3] Manyele, S.V., Zhu, J.-X., Khayat, R.E. and Pärssinen, J.H. (2006) Analysis of the Chaotic Dynamics of a High-Flux CFB Riser Using Solids Concentration Measurements. China Particuology, 4, 136-146. https://doi.org/10.1016/S1672-2515(07)60253-X

[4] de Castilho, G.J. and Cremasco, M.A. (2012) Comparison of Downer and Riser Flows in a Circulating Bed by Means of Optical Fiber Probe Signals Measurement. Procedia Engineering, 42, 295-302. https://doi.org/10.1016/j.proeng.2012.07.420

[5] Kantz, H. and Shreiber, T. (2004) Nonlinear Time Series Analysis. 2nd Edition, Cambridge University Press, Cambridge.

[6] Manyele, S.V., Zhu, J.-X. and Zhang, H. (2003) Analysis of the Microscopic Flow Structure of a CFB Downer Reactor Using Solids Concentration Signals. International Journal of Chemical Reactor Engineering, 1, 1-17. https://doi.org/10.2202/1542-6580.1108

[7] Qiu, G., Ye, J. and Wang, H. (2015) Investigation of Gas-Solid Flow Characteristics in a Circulating Fluidized Bed with Annular Combustion Chamber by Pressure Measurements and CPFD Simulation. Chemical Engineering Science, 134, 433-447. https://doi.org/10.1016/j.ces.2015.05.036

[8] Grassberger, P. and Procaccia, I. (1983) Measurement of Strangeness of the Strange Attractors. Physica, 9D, 189-208.

[9] Singh, P.P. and Handa, H. (2012) Various Synchronization Schemes for Chaotic Dynamical Systems (A Classical Survey). International Journal of Scientific Engineering and Technology, 3, 29-33.

[10] Reagan, A. (2014) Predicting Flow Reversal in a Computational Fluid Dynamics Simulated Thermosyphon Using Data Simulation. Master Thesis, University of Vermont, Burlington.

[11] Sevil, H.E. (2006) On the Predictability of Time Series by Metric Entropy. Master Thesis, Izmir Institute of Technology, İzmir. 
[12] Ding, M., Grebogi, C., Ott, E., Sauer, T. and Yorke, J.A. (1993) Estimating Correlation Dimension from a Chaotic Time Series: When Does Plateau Onset Occur? Physica D, 69, 404-424. https://doi.org/10.1016/0167-2789(93)90103-8

[13] Lai, Y. and Lerner, D. (1998) Effective Scaling Regime for Computing the Correlation Dimension from Chaotic Time Series. Physica D, 115, 1-18. https://doi.org/10.1016/S0167-2789(97)00230-3

[14] Ji, C.C., Zhu, H. and Jiang, W. (2011) A Novel Method to Identify the Scaling Region for Chaotic Time Series Correlation Dimension Calculation. Chinese Science Bulletin, 56, 925-932. https://doi.org/10.1007/s11434-010-4180-6

[15] Shang, P., Li, X. and Kamae, S. (2005) Chaotic Analysis of Traffic Time Series. Chaos, Solitons and Fractals, 25, 121-128.

https://doi.org/10.1016/j.chaos.2004.09.104

[16] Hanias, M.P., Nistazakis, H.E. and Tombras, G.S. (2011) Optoelectronic Chaotic Circuits. In: Sergiyenko, O., Ed., Optoelectronic Devices and Properties, Intech, Shanghai, 631-650. https://doi.org/10.5772/15598

[17] Galka, A., Maaß, T. and Pfister, G. (1998) Estimating the Dimension of High-Dimensional Attractors: A Comparison between Two Algorithms. Physica D, 121, 237-251. https://doi.org/10.1016/S0167-2789(98)00168-7

[18] Park, J.C. (2000) Chaos and Predictability of Internet Transmission Times. Complex Systems, 12, 297-316.

[19] Strozzi, F., Tenrreiro, E.G., Noè, C., Rossi, T., Serati, M. and Comenges, J.Z. (2007) Application of Non-Linear Time Series Analysis Techniques to the Nordic Spot Electricity Market Data. Liuc Papers n. 200, Serie Tecnologia, 11, 1-51.

[20] Li, Q. and Li, K. (2007) Low-Dimensional Chaos of High-Latitude Solar Activity. Astronomical Society of Japan, 59, 983-987.

[21] Kugiumtzis, D. (1996) State Space Reconstruction Parameters in the Analysis of Chaotic Time Series-The Role of the Time Window Length. Physica D: Nonlinear Phenomena, 95, 13-28. https://doi.org/10.1016/0167-2789(96)00054-1

[22] Martins, O.Y., Sadeeq, M.A. and Ahaneku, I.E. (2011) Nonlinear Deterministic Chaos in Benue River Flow Daily Time Sequence. Journal of Water Resource and Protection, 3, 747-757. https://doi.org/10.4236/jwarp.2011.310085

[23] Liang, W.-G. and Zhu, J.-X. (1997) A Core-Annulus Model for the Radial Flow Structure in a Liquid-Solid Circulating Fluidized Bed (LSCFB). Chemical Engineering Journal, 68, 51-62. https://doi.org/10.1016/S1385-8947(97)00065-X 